\title{
Urate as a Marker of Risk and Progression of Neurodegenerative Disease
}

\author{
Sabrina Paganoni ${ }^{1,2,3}$ (D) Michael A. Schwarzschild ${ }^{1,4}$
}

Published online: 19 December 2016

(C) The American Society for Experimental NeuroTherapeutics, Inc. 2016

\begin{abstract}
Urate is a naturally occurring antioxidant whose levels are associated with reduced risk of developing Parkinson's disease (PD) and Alzheimer's disease. Urate levels are also associated with favorable progression in PD, amyotrophic lateral sclerosis, Huntington's disease, and multisystem atrophy. These epidemiological data are consistent with laboratory studies showing that urate exhibits neuroprotective effects by virtue of its antioxidant properties in several preclinical models. This body of evidence supports the hypothesis that urate may represent a shared pathophysiologic mechanism across neurodegenerative diseases. Most importantly, beyond its role as a molecular predictor of disease risk and progression, urate may constitute a novel therapeutic target. Indeed, clinical trials of urate elevation in PD and amyotrophic lateral sclerosis are testing the impact of raising peripheral urate levels on disease outcomes. These studies will contribute to unraveling the neuroprotective potential of urate in human pathology. In parallel, preclinical experiments are deepening our understanding of the molecular pathways that underpin urate's activities. Altogether, these efforts will bring about new insights into the translational potential of urate, its determinants, and its targets and their relevance to neurodegeneration.
\end{abstract}

Sabrina Paganoni

spaganoni@partners.org

1 Harvard Medical School, Department of Neurology, Massachusetts General Hospital, Boston, MA, USA

2 Department of Physical Medicine and Rehabilitation, Spaulding Rehabilitation Hospital, Boston, MA, USA

3 VA Boston Healthcare System, Boston, MA, USA

4 MassGeneral Institute for Neurodegenerative Disease (MIND), Boston, MA, USA
Keywords Biomarker $\cdot$ Risk $\cdot$ Prognosis $\cdot$ Inosine $\cdot$ Oxidative stress

\section{Introduction}

Urate has been gaining momentum as a promising marker of reduced risk and milder progression in several neurodegenerative diseases, most notably Parkinson's disease (PD) and amyotrophic lateral sclerosis (ALS). Interestingly, uratemediated mechanisms may represent a bridge linking diverse neurodegenerative processes. They may also represent an opportunity for synergies among different neurologic diseases along parallel translational paths (Fig. 1). While the biological mechanisms that cause neurodegeneration are not completely understood, they are likely to include damage from oxidative stress, as supported by both autopsy and laboratory studies in several disease models $[1,2]$. These findings provide the rationale for investigating antioxidant systems, such as urate, as potential disease biomarkers and therapeutic targets.

Urate is present in bodily fluids as the anionic form of uric acid (2,6,8-trioxy-purine). It possesses antioxidant properties comparable to those of ascorbate [3], and accounts for most of the antioxidant capacity in human plasma $[4,5]$. Urate is a product of purine metabolism. In rodents, urate is further metabolized to allantoin by urate oxidase (encoded by Uox), while in higher primates urate is the end product of purine metabolism. The difference across species is due to mutations in Uox that arose during evolution and led to the absence of functional urate oxidase in great apes and humans [6]. In humans, urate circulates in blood at high concentrations near the limits of its solubility (accounting for our susceptibility to gout and urolithiasis) [7]. Its blood concentration depends on dietary intake (e.g., meats, seafood, and beer have high purine content) [8], and excretion (which varies based on kidney 


\section{Parkinson's disease}
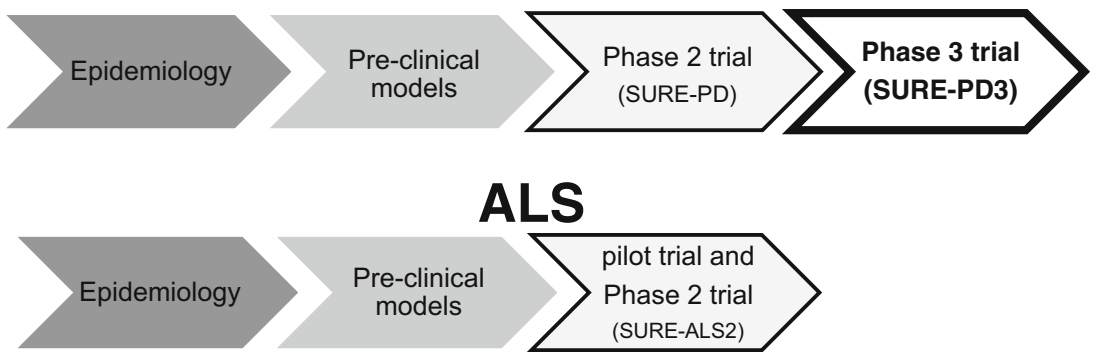

Fig. 1 Translational potential of urate across neurologic diseases. Epidemiologic and laboratory evidence support a neuroprotective role of urate. Urate elevation has been employed in early clinical trials and has rapidly advanced to phase III testing in Parkinson's disease (SUREPD3 trial; NCT02642393; open to enrollment at the time of this publication). Enrollment for SURE-ALS2 is scheduled to begin in early 2017. ALS = amyotrophic lateral sclerosis; PD = Parkinson's disease; SURE-ALS2 = safety of urate elevation in ALS, phase II; SURE$\mathrm{PD}=$ safety of urate elevation in PD; SURE-PD3 = study of urate elevation in PD, phase III

\section{Urate and its Determinants are a Risk Factor for Neurodegenerative Disease}

If urate were protective against neurodegeneration, one might expect its levels to be low in patients and to correlate with disease risk. Indeed, plasma or serum urate levels are lower in people with PD [44, 45] and ALS [46, 47] than healthy controls. Further, in postmortem substantia nigra tissue, urate levels are lower in patients with PD than in age-matched controls [12].

In longitudinal cohort studies, urate levels correlate with risk of developing PD later in life, with higher levels being associated with reduced disease risk [13-17]. History of gout is associated with reduced risk of developing either PD [48] or Alzheimer's disease [21]. Urate level determinants also modify susceptibility to PD. Thus, dietary factors that contribute to higher plasma urate are associated with a lower risk of PD (top $v s$ bottom quintile: relative risk $0.47, p=0.0008$ ), after adjustment for potential confounders [18], and genetic variability in genes known to influence urate levels is associated with PD risk $[19,20]$.

Table 1 Evidence for the roles of urate in neuroprotection

\begin{tabular}{lllll}
\hline Type of evidence & & PD & ALS & Others \\
\hline Epidemiologic & Risk & $\begin{array}{l}\text { Inverse association } \\
{[13-20]}\end{array}$ & & Inverse association with AD risk [21] \\
$\begin{array}{l}\text { (urate levels, urate genetic } \\
\text { determinants, diet) }\end{array}$ & Progression & Inverse association [22-24] & $\begin{array}{l}\text { Inverse association [25-28] } \\
\text { No association [29, 30] }\end{array}$ & $\begin{array}{l}\text { Inverse association in HD, MSA, MCI [31-33] } \\
\text { Laboratory }\end{array}$ \\
& In vitro & Neuroprotective [34-36] & & $\begin{array}{l}\text { Neuroprotective in cultured } \\
\text { spinal cord neurons [37] }\end{array}$ \\
& In vivo & Neuroprotective [35] & $\begin{array}{l}\text { Neuroprotective in SCI, brain } \\
\text { ischemia/stroke, MS [38-41] } \\
\text { URICO-ICTUS (phase IIb/III trial in stroke) }\end{array}$ \\
& & SURE-PD (phase II trial) [10] & [42, 43]
\end{tabular}

$\mathrm{PD}=$ Parkinson's disease; $\mathrm{ALS}=$ amyotrophic lateral sclerosis; $\mathrm{AD}=$ Alzheimer's disease $\mathrm{HD}=$ Huntington's disease; MSA = multiple system atrophy; $\mathrm{MCI}=$ mild cognitive impairment; $\mathrm{SCI}=$ spinal cord injury; $\mathrm{MS}=$ multiple sclerosis 
Urate and its Determinants are Molecular Predictors of Disease Progression in Neurodegenerative Diseases

The strongest evidence for urate as a molecular predictor of disease progression comes from PD studies. In the PRECEPT clinical trial, the risk of PD disability progressing to the need for dopaminergic therapy among those in the highest quintile of serum urate concentration at baseline was half that of the lowest quintile [22]. Similarly, in the DATATOP clinical trial, risk of PD progression was reduced by $18 \%$ for each $1.5 \mathrm{mg} / \mathrm{dl}$ increase in serum urate concentration, with a similar inverse correlation between CSF urate and progression [23]. A causal link between urate levels and PD outcomes is strengthened by the finding that genetic determinants of urate levels also predict PD disease progression [24]. Thus, urate and its determinants are robust biomarkers of $\mathrm{PD}$ at different points in the disease process (Fig. 2).

A link between urate levels and disease outcomes has also been suggested across a range of other neurodegenerative diseases, raising the hypothesis of a broad neuroprotective effect of urate on multiple CNS neuronal populations. Higher urate levels are associated with slower clinical progression in Huntington's disease, multiple system atrophy, and mild cognitive impairment [29-31]. In ALS, most [25-28] but not all $[32,33]$ studies found urate to represent a prognostic factor for survival. The reasons for these conflicting results in ALS are not completely clear and may be related to methodological differences, variable sample size, and a modest effect size.

\section{Urate is Neuroprotective in Several Preclinical Models of Neurodegeneration}

Despite their statistical strength and reproducibility, these epidemiologic studies cannot address the question of whether urate is directly implicated in disease pathogenesis or may simply represent a byproduct of disease mechanisms. Several experimental studies support the former hypothesis and suggest that urate may have a direct impact on neuroprotective mechanisms across a range of neurodegenerative processes.

In vitro, in PD models, urate prevents spontaneous degeneration of cultured nigral neurons, as well as dopaminergic cell death induced by oxidative and mitochondrial toxins $[34,36]$. In vivo, genetic manipulation of urate oxidase and resulting increased concentrations of urate in the CNS led to improved phenotype and histopathologic findings in PD mouse models $[35,49]$. These data substantiate earlier findings in PC12 cells, in which urate blocked cell death and oxidative damage induced by either dopamine [50] or 6hydroxydopamine [51]. Similarly, in a rotenone toxicity model, urate prevented death of dopaminergic cells [52]. At physiologically relevant concentrations, urate was also shown to enhance function and survival of dopaminergic neurons in primary cultures of rat ventral mesencephalon [53]. Interestingly, urate-mediated effects may require its accumulation in astrocytes, suggesting a noncell-autonomous mechanism for urate's neuroprotective activity [36, 37]. The mechanisms underlying urate's neuroprotective effects are not completely clear, though recent in vitro studies have begun to shed light on urate's targets. These studies showed that urate treatment results in nuclear translocation of the nuclear factor (erythroid-derived 2)-like 2 protein, a master regulator of the response to oxidative stress, and transcriptional activation of its key target genes in primary astrocytic cultures $[54,55]$. In this model, urate treatment led to a marked increase in glutathione synthesis and release [54]. Additional ongoing studies into the downstream effects of urate may identify novel therapeutic targets for neurodegenerative diseases.

Urate confers protection in various models of neurotoxicity. Urate protected cultured spinal cord neurons from glutamate toxicity [37] and was neuroprotective in models of spinal cord injury, multiple sclerosis, brain injury, and stroke [38-41, 56]. This growing literature suggests a potential neuroprotective role for urate beyond PD. The effects of urate in preclinical models of ALS are currently under investigation.
Fig. 2 Biomarker properties of urate across the timeline of Parkinson's disease (PD). Several studies support urate or its determinants (dietary or genetic) as a biomarker of PD at different points in the PD stage, which refers to Hoehn and Yahr scale stage

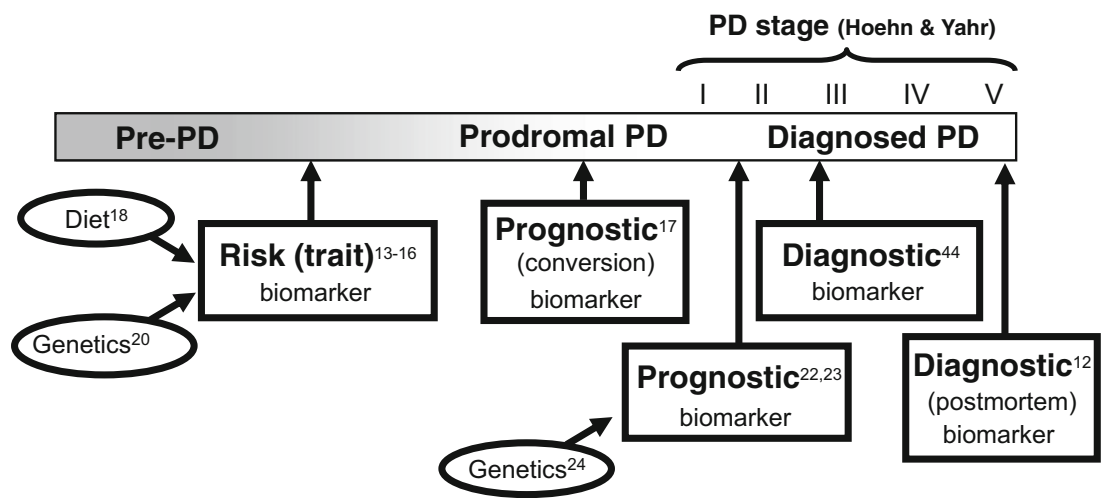




\section{Early Clinical Trial Evidence Related to Urate Elevation as a Therapeutic Target}

Based on the strong preclinical and epidemiologic evidence supporting a neuroprotective role for urate in the CNS, recent clinical trials by our group and others have begun to examine the feasibility of urate manipulation in a clinical setting with promising results $[10,42]$. The Safety of Urate Elevation in Parkinson's Disease (SURE-PD) trial was a phase II randomized, double-blind, placebo-controlled, dose-ranging trial of urate-elevating inosine in PD [10]. While urate is rapidly degraded by intestinal flora when taken by mouth, its precursor inosine when taken orally is rapidly metabolized to urate, and is available as an over-the-counter supplement. Results from the SURE-PD trial demonstrated that inosine supplementation is clinically safe and tolerable for up to 24 months and leads to a dose-dependent increase in serum and CSF urate levels in patients with PD. Although the study was not powered to test efficacy, secondary analyses indicated nonfutility of inosine treatment for slowing disability [10]. Of note, no instances of gout occurred during the study, but $6 \%$ of study participants who were randomized to active drug developed kidney stones. Given these promising results, urate elevation is being pursued as a potential disease-modifying treatment for PD in a phase III study (SURE-PD3 trial; NCT02642393; open to enrollment as of August 2016).

While urate is a mature target in PD and experience on inosine pharmacology is rapidly accumulating, urate manipulation is a relatively new target for ALS and other neurologic diseases. A pilot, open-label study of urate elevation in ALS (NCT02288091) was recently completed demonstrating the feasibility of using inosine to elevate urate levels in patients with ALS (SP, personal communication). A follow-up, multicenter, biomarker-driven, placebo-controlled phase II study (SURE-ALS2) is in the planning stages and is scheduled to begin enrollment in early 2017. Encouraging clinical results were also recently obtained by the Safety and Efficacy of Uric Acid in Patients with Acute Stroke (URICO-ICTUS) study: a phase IIb/III randomized, double-blind trial of intravenous urate administration within a few hours of stroke onset [42, 43]. In this study, the combination of urate treatment with thrombolysis in acute ischemic stroke resulted in improved clinical outcomes in women but not men. Safety remains an important focus of these studies given associations of higher urate and disorders other than gout and uric acid kidney stones, including cardiovascular and metabolic disorders [57].

\section{Conclusions}

Epidemiologic evidence strongly supports urate as a predictor of disease risk and progression in PD (Fig. 2), with data in PD models demonstrating that urate can confer neuroprotection against oxidative stress-induced neuronal death. These findings have substantial therapeutic implications and justify direct translation to clinical trials such as the ongoing phase III study of urate elevation in PD (SURE-PD3 trial; NCT02642393). A growing body of literature supports similar roles for urate in ALS where early clinical trials are underway to begin to test the feasibility and safety of urate elevation as a potential disease-modifying intervention. In parallel, preclinical research is beginning to unravel the downstream mechanisms that mediate urate's effects. These studies may unravel novel therapeutic targets that may be of relevance for several neurodegenerative diseases.

Acknowledgements S.P. has received funding from the National Institutes of Health (NIH; Career Development Award 2K12HD001097-16), Target ALS, the Salah Foundation, the Spastic Paraplegia Foundation, the ALS Association, ALS Finding a Cure, and the MGH ALS Therapy Fund. M.S. has received grants from the NIH, the Michael J. Fox Foundation, the Parkinson's Disease Foundation, the Department of Defense, the Hoffman Foundation, and Target ALS; M.S. has received personal fees from Biotie Therapeutics.

Required Author Forms Disclosure forms provided by the authors are available with the online version of this article.

\section{References}

1. D'Amico E, Factor-Litvak P, Santella RM, Mitsumoto H. Clinical perspective on oxidative stress in sporadic amyotrophic lateral sclerosis. Free Radic Biol Med 2013;65:509-527.

2. Henchcliffe C, Beal MF. Mitochondrial biology and oxidative stress in Parkinson disease pathogenesis. Nat Clin Pract Neurol 2008;4:600-609.

3. Proctor P. Similar functions of uric acid and ascorbate in man? Nature 1970;228:868.

4. Yeum KJ, Russell RM, Krinsky NI, Aldini G. Biomarkers of antioxidant capacity in the hydrophilic and lipophilic compartments of human plasma. Arch Biochem Biophys 2004;430:97-103.

5. Fabbrini E, Serafini M, Colic Baric I, Hazen SL, Klein S. Effect of plasma uric acid on antioxidant capacity, oxidative stress, and insulin sensitivity in obese subjects. Diabetes 2014;63:976-981.

6. Johnson RJ, Titte S, Cade JR, Rideout BA, Oliver WJ. Uric acid, evolution and primitive cultures. Semin Nephrol 2005;25:3-8.

7. Cipriani S, Chen X, Schwarzschild MA. Urate: a novel biomarker of Parkinson's disease risk, diagnosis and prognosis. Biomark Med 2010;4:701-712.

8. Torralba KD, De Jesus E, Rachabattula S. The interplay between diet, urate transporters and the risk for gout and hyperuricemia: current and future directions. Int J Rheum Dis 2012;15:499-506.

9. Vitart V, Rudan I, Hayward C, et al. SLC2A9 is a newly identified urate transporter influencing serum urate concentration, urate excretion and gout. Nat Genet 2008;40:437-442.

10. Schwarzschild MA, Ascherio A, Beal MF, et al. Inosine to increase serum and cerebrospinal fluid urate in Parkinson disease: a randomized clinical trial. JAMA Neurol 2014;71:141-150.

11. Ames BN, Cathcart R, Schwiers E, Hochstein P. Uric acid provides an antioxidant defense in humans against oxidant- and radicalcaused aging and cancer: a hypothesis. Proc Natl Acad Sci U S A 1981;78:6858-6862. 
12. Church WH, Ward VL. Uric acid is reduced in the substantia nigra in Parkinson's disease: effect on dopamine oxidation. Brain Res Bull 1994;33:419-425.

13. Davis JW, Grandinetti A, Waslien CI, Ross GW, White LR, Morens DM. Observations on serum uric acid levels and the risk of idiopathic Parkinson's disease. Am J Epidemiol 1996;144:480-484.

14. Weisskopf MG, O'Reilly E, Chen H, Schwarzschild MA, Ascherio A. Plasma urate and risk of Parkinson's disease. Am J Epidemiol 2007; 166:561-567.

15. Chen H, Mosley TH, Alonso A, Huang X. Plasma urate and Parkinson's disease in the Atherosclerosis Risk in Communities (ARIC) study. Am J Epidemiol 2009;169:1064-1069

16. de Lau LM, Koudstaal PJ, Hofman A, Breteler MM. Serum uric acid levels and the risk of Parkinson disease. Ann Neurol 2005;58: 797-800.

17. Uribe-San Martin R, Venegas Francke P, Lopez Illanes F, et al. Plasma urate in REM sleep behavior disorder. Mov Disord 2013;28:1150-1151.

18. Gao X, Chen H, Choi HK, Curhan G, SchwarzschildMA, Ascherio A. Diet, urate, and Parkinson's disease risk in men. Am J Epidemiol 2008; 167:831-838.

19. Facheris MF, Hicks AA, Minelli C, et al. Variation in the uric acid transporter gene SLC2A9 and its association with AAO of Parkinson's disease. J Mol Neurosci 2011;43:246-250.

20. Gonzalez-Aramburu I, Sanchez-Juan P, Jesus S, et al. Genetic variability related to serum uric acid concentration and risk of Parkinson's disease. Mov Disord 2013;28:1737-1740.

21. Lu N, DubreuilM, Zhang Y, et al. Gout and the risk of Alzheimer's disease: a population-based, BMI-matched cohort study. Ann Rheum Dis 2016;75:547-551.

22. Schwarzschild MA, Schwid SR, Marek K, et al. Serum urate as a predictor of clinical and radiographic progression in Parkinson disease. Arch Neurol 2008;65:716-723.

23. Ascherio A, LeWitt PA, Xu K, et al. Urate as a predictor of the rate of clinical decline in Parkinson disease. Arch Neurol 2009;66: $1460-1468$.

24. Simon KC, Eberly S, Gao X, et al. Mendelian randomization of serum urate and parkinson disease progression. Ann Neurol 2014; 76:862-868

25. Paganoni S, Zhang M, Quiroz Zarate A, et al. Uric acid levels predict survival in men with amyotrophic lateral sclerosis. J Neurol 2012;259:1923-1928.

26. Abraham A, Drory VE. Influence of serum uric acid levels on prognosis and survival in amyotrophic lateral sclerosis: a metaanalysis. J Neurol 2014;261:1133-1138.

27. Atassi N, Berry J, Shui A, et al. The PRO-ACT database: design, initial analyses, and predictive features. Neurology 2014;83:17191725 .

28. Kuffner R, Zach N, Norel R, et al. Crowdsourced analysis of clinical trial data to predict amyotrophic lateral sclerosis progression. Nat Biotechnol 2015;33:51-57.

29. Zheng Z, Guo X, Wei Q, et al. Serum uric acid level is associated with the prevalence but not with survival of amyotrophic lateral sclerosis in a Chinese population. Metab Brain Dis 2014;29:771775.

30. Chio A, Calvo A, Bovio G, et al. Amyotrophic lateral sclerosis outcome measures and the role of albumin and creatinine: a population-based study. JAMA Neurol 2014;71:1134-1142.

31. Auinger P, Kieburtz K, McDermott MP. The relationship between uric acid levels and Huntington's disease progression. Mov Disord 2010;25:224-228.

32. Irizarry MC, Raman R, Schwarzschild MA, et al. Plasma urate and progression of mild cognitive impairment. Neurodegener Dis 2009;6:23-28.
33. Lee JE, Song SK, SohnYH, Lee PH.Uric acid as a potential disease modifier in patients with multiple system atrophy. Mov Disord 2011;26:1533-1536.

34. Cipriani S, Desjardins CA, Burdett TC, Xu Y, Xu K, Schwarzschild MA. Urate and its transgenic depletion modulate neuronal vulnerability in a cellular model of Parkinson's disease. PLOS ONE 2012;7:e37331.

35. ChenX, Burdett TC, Desjardins CA, et al. Disrupted and transgenic urate oxidase alter urate and dopaminergic neurodegeneration. Proc Natl Acad Sci U S A 2013;110:300-305.

36. Cipriani S, Desjardins CA, Burdett TC, Xu Y, Xu K, Schwarzschild MA. Protection of dopaminergic cells by urate requires its accumulation in astrocytes. J Neurochem 2012;123:172-181.

37. Du Y, Chen CP, Tseng CY, Eisenberg Y, Firestein BL. Astrogliamediated effects of uric acid to protect spinal cord neurons from glutamate toxicity. Glia 2007;55:463-472.

38. Yu ZF, Bruce-Keller AJ, Goodman Y, Mattson MP. Uric acid protects neurons against excitotoxic and metabolic insults in cell culture, and against focal ischemic brain injury in vivo. J Neurosci Res 1998;53:613-625.

39. Scott GS, Cuzzocrea S, Genovese T, Koprowski H, Hooper DC. Uric acid protects against secondary damage after spinal cord injury. Proc Natl Acad Sci U S A 2005;102:3483-3488.

40. Romanos E, Planas AM, Amaro S, Chamorro A. Uric acid reduces brain damage and improves the benefits of rt-PA in a rat model of thromboembolic stroke. J Cereb Blood FlowMetab 2007;27:14-20.

41. Kean RB, Spitsin SV, Mikheeva T, Scott GS, Hooper DC. The peroxynitrite scavenger uric acid prevents inflammatory cell invasion into the central nervous system in experimental allergic encephalomyelitis through maintenance of blood-central nervous system barrier integrity. J Immunol 2000;165:6511-6518.

42. Chamorro A, Amaro S, CastellanosM, et al. Safety and efficacy of uric acid in patients with acute stroke (URICO-ICTUS): a randomised, double-blind phase $2 \mathrm{~b} / 3$ trial. Lancet Neurol 2014;13:453-460.

43. Llull L, Laredo C, Renu A, et al. Uric acid therapy improves clinical outcome in women with acute ischemic stroke. Stroke 2015;46: 2162-2167.

44. Annanmaki T, Muuronen A,Murros K. Low plasma uric acid level in Parkinson's disease. Mov Disord 2007;22:1133-1137.

45. BogdanovM,MatsonWR, Wang L, et al.Metabolomic profiling to develop blood biomarkers for Parkinson's disease. Brain 2008;131:389-396.

46. Lawton KA, Brown MV, Alexander D, et al. Plasma metabolomic biomarker panel to distinguish patients with amyotrophic lateral sclerosis from disease mimics. Amyotroph Lateral Scler Frontotemporal Degener 2014;15:362-370.

47. Keizman D, Ish-ShalomM, Berliner S, et al. Low uric acid levels in serum of patients with ALS: further evidence for oxidative stress? J Neurol Sci 2009;285:95-99.

48. Alonso A, Rodriguez LA, Logroscino G, Hernan MA. Gout and risk of Parkinson disease: a prospective study. Neurology 2007;69: 1696-1700.

49. Gong L, Zhang QL, Zhang N, et al. Neuroprotection by urate on 6OHDA-lesioned rat model of Parkinson's disease: linking to Akt/ GSK3beta signaling pathway. J Neurochem 2012;123:876-885.

50. Jones DC, Gunasekar PG, Borowitz JL, Isom GE. Dopamineinduced apoptosis is mediated by oxidative stress and Is enhanced by cyanide in differentiated PC12 cells. J Neurochem 2000;74:2296-2304

51. Zhu TG, Wang XX, Luo WF, et al. Protective effects of urate against 6-OHDA-induced cell injury in PC12 cells through antioxidant action. Neurosci Lett 2012;506:175-179.

52. Duan W, Ladenheim B, Cutler RG, Kruman, II, Cadet JL, Mattson MP. Dietary folate deficiency and elevated homocysteine levels 
endanger dopaminergic neurons in models of Parkinson's disease. $\mathrm{J}$ Neurochem 2002;80:101-110.

53. Guerreiro S, Ponceau A, Toulorge D, et al. Protection of midbrain dopaminergic neurons by the end-product of purine metabolism uric acid: potentiation by low-level depolarization. J Neurochem 2009;109:1118-1128.

54. Bakshi R, Zhang H, Logan R, et al. Neuroprotective effects of urate are mediated by augmenting astrocytic glutathione synthesis and release. Neurobiol Dis 2015;82:574-579.
55. Zhang N, Shu HY, Huang T, et al. Nrf2 signaling contributes to the neuroprotective effects of urate against 6-OHDA toxicity. PLOS ONE 2014;9:e100286.

56. Onetti Y, Dantas AP, Perez B, et al. Middle cerebral artery remodeling following transient brain ischemia is linked to early postischemic hyperemia: a target of uric acid treatment. Am J Physiol Heart Circ Physiol 2015;308:H862-H874.

57. Johnson RJ. Why focus on uric acid? Curr Med Res Opin 2015;31(Suppl. 2):3-7. 